\title{
A Model of Calcium Homeostasis Linked with Electrostrictive Energy
}

\section{Suman Halder ${ }^{1 *}$ and Abhay Shankar Chakraborty ${ }^{2}$}

'ST. Thomas College of Engineering \& Technology, Kolkata-23

${ }^{2}$ Raja Bazar Science College, Kolkata

\begin{abstract}
The present paper describes how calcium homeostasis is related with electrostrictive energy linked with $\mathrm{pH}$ homeostasis. The proposed model incorporates electrostrictive energy as input and bone calcium level as output. The model is also comprised of $\mathrm{pH}$ homeostasis, antiporter homeostasis, PTH homeostasis and calcium homeostasis. Electrostrictive energy in E. Coli plays very crucial role for homeostasis of bone calcium. As we know that bone calcium plays very important roles, which are linked with fundamental biological processes involving muscle contraction including the cardiac muscles, which can prevent myocardial infarction during heart attack. Tight regulation of the extracellular fluid (ECF) calcium concentration is maintained through the action of calcium sensitive cells. The precise control of calcium ion in extracellular fluids is of great importance for maintaining the health of individuals. The calcium homeostasis is linked with parathyroid hormone and VDR depending on the level of calcium in ECF and formation of osteoblast cells. Also the homeostasis of intracellular calcium ([Ca++]i) and $\mathrm{pH}(\mathrm{pHi})$ is important in the cell's ability to respond to growth factors, to initiate differentiation and proliferation, and to maintain normal metabolic pathways.

This model has been simulated by using MATLAB 6.5 in order to facilitate comprehensive understanding of complex electrostrictive energy phenomena concomitant with capacitance relaxation phenomena associated with cancer.

It has been revealed from the model that, more is the change of electrostrictive energy more will be the reduction of formation of osteoblast cells and decreased production of bone calcium. Electrostrictive energy linked with $\mathrm{pH}$ homeostasis affects the formation of osteoblast cells. The osteoblast cell lineage is responsible for bone development, growth, and remodeling. Variety of hormones and growth factors regulates the development of rapidly dividing osteoprogenitor cells into highly differentiated bone-forming osteoblasts. Activation of E Coli. due to electrostrictive energy reduces formation of osteoblast cells which will affect calcium homeostasis.
\end{abstract}

Keywords: Electrostrictive energy; pH homeostasis; Calcium homeostasis; Osteoblast cell lineage

\section{Introduction}

As stable physiologic systemic $\mathrm{pH}$ is critical for the survival of mammals. Homeostasis of the intracellular hydrogen ion concentration is pivotal to the maintenance of cell function and viability, but $\mathrm{pH}$ fluctuations are present in both intracellular and extracellular compartments in normal physiological and pathophysiological conditions [1-4]. Bone cells, both osteoblasts and osteoblasts, respond to $\mathrm{pH}$ changes in the physiological range, the sensors that can mediate these $\mathrm{pH}$ changes are electrostrictive energy variation due to capacitance relaxation [5].

Because of the importance of $\mathrm{pH}$ homeostasis in bone and the current uncertainty about the mechanisms by which intracellular $\mathrm{pH}$ (pH1) is regulated in this tissue, the roles of cytosolic free $\mathrm{Ca}^{2+}$ concentrations $\left(\left[\mathrm{Ca}^{2+}\right]\right.$,) have been investigated and protein kinase $\mathrm{C}$ on the activation of $\mathrm{Na}^{+} / \mathrm{H}^{+}$exchange in human osteoblast-like SaOS-2 cells. [ $\mathrm{Ca}^{2+}$, and $\mathrm{pH}$, were measured using Fura-2 and 2'7'-bis-(2-carboxyethyl)-5(6)carboxyfluorescein (BCECF) respectively. Addition of ionomycin in $\mathrm{Ca}^{2+}$-containing buffer did not cause a rise in basal $\mathrm{pH}$, however, addition of the phorbol ester phorbol 12-myristate 13-acetate (PMA) did cause a slowly developing rise in resting $\mathrm{pH}$, of $0.14+0.02$ unit over 4-5 min. Nigericin, a $\mathrm{K}^{+} / \mathrm{H}^{+}$ionophore, caused an abrupt fall in $\mathrm{pH}$, to $6.70+0.07$ units. In nigericin-pretreated cells, PMA caused a rapid rise in $\mathrm{pH}$ without changing the $\left[\mathrm{Ca}^{2+}\right][6]$. In acidified cells, ionomycin increased $\left[\mathrm{Ca}^{2+}\right]$ and $\mathrm{pH}$ in a parallel concentration-dependent $(30-500$ $\mathrm{nM}$ ) manner. This action of ionomycin occurred in both the presence and the nominal absence of extracellular $\mathrm{Ca}^{2+}[7]$.
The algorithm for calculating ionized calcium at $\mathrm{pH} 7.40$ may be deduced from the following equation:

$$
\left[\mathrm{Ca}^{2+}\right](\mathrm{pH} 7.40)=\left[\mathrm{Ca}^{2+}\right]^{\star} 10^{\mathrm{b}(7.40-\mathrm{pH})}
$$

Where $\left[\mathrm{Ca}^{2+}\right]$ is the measured concentration of ionized calcium, $\left[\mathrm{Ca}^{2+}\right]$ ( $\left.\mathrm{pH} 7.40\right)$ is the calculated standard ionized calcium at $\mathrm{pH}$ 7.40, and $b$ is the experimentally found slope $\left(\operatorname{dlog} 10\left[\mathrm{Ca}^{2+}\right] / \mathrm{dpH}\right)$ for the linear relation between $\log 10\left[\mathrm{Ca}^{2+}\right]$ and $\mathrm{pH}$ for whole blood, plasma, or serum [8].

The electrostrictive phenomenon is due to the composite dielectric property of the cancer cell concomitant with the capacitance relaxation phenomena [5]. It is to be noted that $\mathrm{pH}$ homeostats in cancer cells are directly linked with the gene homeostat in a novel way so far as dephosphorylation of the oncogenes is concerned. This phenomenon in respect of $\mathrm{pH}$ homeostasis has been correlated with capacitance relaxation phenomenon and electrostrictive energy during $\mathrm{pH}$ mediated signal transduction phase [5,9].

The calcium homeostasis, the phosphate homeostasis, formation

*Corresponding author: Suman Halder, ST. Thomas College of Engneering \& Technology, Kolkata-23, E-mail: sum_hal@yahoo.co.in

Received October 03, 2011; Accepted November 21, 2011; Published November 25, 2011

Citation: Halder S, Shankar Chakraborty A (2011) A Model of Calcium Homeostasis Linked with Electrostrictive Energy. J Health Med Informat 3:107. doi:10.4172/2157-7420.1000107

Copyright: ( 2011 Halder S, et al. This is an open-access article distributed unde the terms of the Creative Commons Attribution License, which permits unrestricted use, distribution, and reproduction in any medium, provided the original author and source are credited. 
of bone and teeth, and regulation of vitamin $\mathrm{D}$, parathyroid hormone (PTH), and calcitonin are all closely intertwined. Extracellular calcium ion concentration is determined by the interplay of calcium absorption from intestine, renal excretion of calcium, and bone uptake and release of calcium, each of which is regulated by PTH, calcitonin. Precise control of extracellular calcium is essential because calcium plays a key role in many physiologic processes, including contraction of skeletal, cardiac, and smooth muscles; blood clotting; and transmission of nerve pulses etc $[6,10]$.

PTH is derived from parathyroid, which are of entodermal origin, are synthesized as pre-pro parathyroid hormones as initial transitional product synthesized on ribosome. PTH is composed of a straight chain of polypeptide consisting of 84 -amino acids. The role of parathyroid hormone (PTH) is to regulate the calcium and phosphate level in extracellular fluid (ECF) concomitant with the proliferation of a high affinity cellular receptor known as VDR $[6,10,11]$.

VDR is a high affinity cellular receptor induced by Vitamin D, 1,25-dihydroxyvitamin $\mathrm{D}_{3}\left[1,25(\mathrm{OH})_{2} \mathrm{D}_{3}\right]$. The mechanism by which phosphorous controls the production of VDR is still unclear but it appears to be independent of PTH and changes in serum calcium. The major regulator of VDR is PTH $[6,10,11]$.

PTH enhances bone reabsorption and liberates both calcium and phosphate from the skeleton. PTH also can enhance calcium reabsorption in the kidney while at the same time inhibit phosphate reabsorption producing phosphaturia. Hypocalcemia and PTH can both stimulate the production of VDR which in turn initiates intestinal calcium and to lesser extent phosphate reabsorption. The effect of suppressing PTH and VDR diminish skeletal calcium release, intestinal calcium reabsorption and renal calcium reabsorption and restore the elevated ECF calcium to normal $[1,10]$. The binding of calcium with protein lead to hyperalbuminemia and hypoalbuminemia depending on the calcium present in ECF [10].

PTH plays a vital role in bone metabolism and modulates the expression of several genes characteristic of the osteoblast phenotype. Binding of PTH to its receptor causes activation of adenylate cyclase, the enzyme responsible for the formation of cAMP. The cAMP pathway is further transduced when cAMP binds to protein kinase $\mathrm{A}$, which in its activated state phosphorylates target molecules in the cytosol. A complex mechanism maintaining the homeostasis of intracellular cAMP consists of cAMP-generating and -inactivating mechanisms [1$3]$.

PTH exerts its effects on mineral homeostasis by influencing the movement of calcium and phosphate into and out of the exchangeable mineral compartment of bone and by regulating mineral handling and 1,25- dihydroxy vitamin D3 formation by the kidney. The hormone acts on these tissues at least in part by stimulating adenylate cyclase in the target cell membrane to increase intracellular cAMP levels (1). PTH increases the cAMP content of cultured murine (1) and hicken (2) bone cells, and PTH receptors have been characterized in bovine $(3,4)$, avian (5), canine (6), and human (6) kidney membranes [1,10].

Low extracellular calcium $\left(\mathrm{Ca}^{2+}\right)$ promotes release of PTH, which acts on multiple organs to maintain overall $\mathrm{Ca}^{2+}$ balance. In the distal part of the nephron, PTH stimulates active $\mathrm{Ca}^{2+}$ reabsorption via the adenylyl cyclase-cAMP-protein kinase A (PKA) pathway, but the molecular target of this pathway is unknown. The transient receptor potential vanilloid 5 (TRPV5) channel constitutes the luminal gate for $\mathrm{Ca}^{2+}$ entry in the distal convoluted tubule and has several putative PKA phosphorylation sites. Here, we investigated the effect of PTH-induced cAMP signaling on TRPV5 activity. Using fluorescence resonance energy transfer, we studied cAMP and $\mathrm{Ca}^{2+}$ dynamics during PTH stimulation of HEK293 cells that co expressed the PTH receptor and TRPV5. PTH increased cAMP levels, followed by a rise in TRPV5mediated $\mathrm{Ca}^{2+}$ influx. PTH (1 to 31 ) and forskolin, which activate the cAMP pathway, mimicked the stimulation of TRPV5 activity. Remarkably, TRPV5 activation was limited to conditions of strong intracellular $\mathrm{Ca}^{2+}$ buffering $[2,10]$.

The production of cAMP by G protein-coupled receptors (GPCRs) and its termination are currently thought to occur exclusively at the plasma membrane of cells. Under existing models of receptor regulation, this signal is primarily restricted by desensitization of the receptors through their binding to $\beta$-arrestins. However, this paradigm is not consistent with recent observations that the parathyroid hormone receptor type 1 (PTHR) continues to stimulate cAMP production even after receptor internalization, as $\beta$-arrestins are known to rapidly bind and internalize activated PTHR. Here we show that binding to $\beta$-arrestin1 prolongs rather than terminates the generation of cAMP by PTHR, and that cAMP generation correlates with the persistence of arrestin-receptor complexes on endosomes. PTHR signaling is instead turned off by the retromer complex, which regulates the movement of internalized receptor from endosomes to the Golgi apparatus. Thus, binding by the retromer complex regulates the sustained generation of cAMP triggered by an internalized GPCR $[10,12]$.

Serum calcium level plays a major role in calcium homeostasis. Experimental studies have revealed that the increase of calcium ion in serum is due to the decrease of phosphate ion. However, the percentage changes of calcium and phosphate ions in serum are almost equal according to mass law equation. Moreover the experimental studies have shown the percentage change in PTH concomitant with calcium and phosphate homeostasis is almost negligible. Calcium plays a very important role, which are linked with fundamental biological processes involving muscle contraction including the cardiac muscles, which can prevent myocardial infarction during heart attack. Tight regulation of the extracellular fluid (ECF) calcium concentration is maintained through the action of calcium sensitive cells. The precise control of calcium ion in extracellular fluids is of great importance for maintaining the health of individuals. The calcium homeostasis is linked with parathyroid hormone and VDR depending on the level of calcium in ECF. The binding of calcium with protein lead to hyperalbuminemia and hypoalbuminemia depending on the calcium present in ECF [11].

The renal effects of parathyroid hormone (PTH) include a decreased rate of acidification by the proximal tubule. To determine whether this effect represented a PTH action on the $\mathrm{Na}^{+}-\mathrm{H}^{+}$antiporter, we investigated the effect of PTH on the established opossum kidney (OK) cell line. This cell line retains several features characteristic of proximal tubule cells, including an amiloride-sensitive $\mathrm{Na}^{+}-\mathrm{H}^{+}$antiporter and high-affinity PTH receptors with a coupled cAMP response [13].

The development of rapidly dividing osteoprogenitor cells into highly differentiated bone-forming osteoblasts is regulated by variety of hormones and growth factors [1-3].

It has been investigated that $\mathrm{BI}-1$, an acidic $\mathrm{pH}$-dependent $\mathrm{Ca}(2+)$ 
channel in osteoblasts causes acidic $\mathrm{pH}$-associated cell death. BI-1 knock-down causes in inhibition of acidic $\mathrm{pH}$-induced release of proinflammatory cytokines, including IL-1 $\beta$, IL-6, and TNF- $\alpha$ which will result differentiation of bone marrow stem cells into human osteoblasts, gives increased expression of BI-1 mRNA and protein [14].

It is well established that ionized calcium in blood is closely related to acid-base quantities. The reason for an increase in ionized calcium accompanying a decrease in $\mathrm{pH}$ is partly the decreased protein binding of calcium at lower $\mathrm{pH}$ and partly a change in the fraction of calcium bound to complexing anions.

\section{Modeling}

$\mathrm{pH}$ homeostasis in cancer cell involves electrostrictive energy as input and oxidant/antioxidant ratio as the output [5]. As the results revealed, more the $\mathrm{pH}$ become basic, less will be the oxidant/antioxidant ratio [5]. As we see that antiporters maintain alkaline environment where as lipid peroxidation initiated by antiporters maintains acidic $\mathrm{pH}$ homeostasis of the fluid of the E. coli archaeabacteria [5]. Then we can say that reduction of oxidant/antioxidant ratio will would cause decrement of $\mathrm{Na}^{+} / \mathrm{H}^{+}$antiporters. Now osteoblast cell linage is dependent on $\mathrm{Na}^{+} / \mathrm{H}^{+}$antiporter concomitant with PKC mediated pathway $[13,15]$. As injection of PTH causes rapid increase in activity of osteoclasts therefore PTH will cause more formation of osteoblast cells [16]. So we can say that more the reduction of antiporter, more will be the decrement of osteoblast progenitor cell linage. So we can say that more the basic is $\mathrm{pH}$, more will be reduction of osteoblast cell formation. This will cause decrement of high-affinity PTH receptors with a coupled cAMP response [13]. Therefore PTH level for calcium homeostasis will be decreased. This will result decreased level of bone calcium.

The block diagram representation of the proposed model is given below in Figure 1.

The explanation of each of the homeostasis is outlined below:

First of all the $\mathrm{pH}$ homeostasis in cancer cell is governed by the

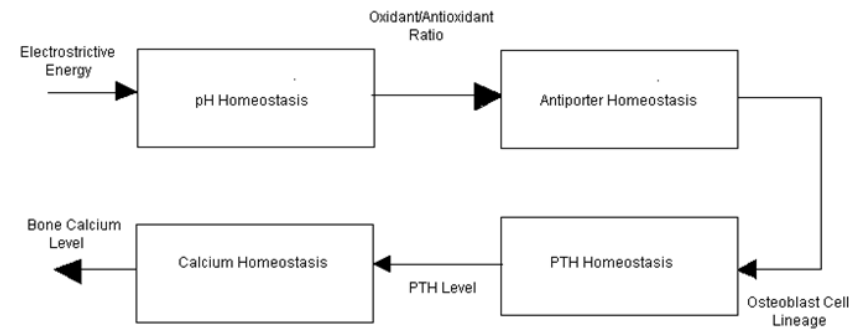

Figure 1: Block diagram of proposed model.

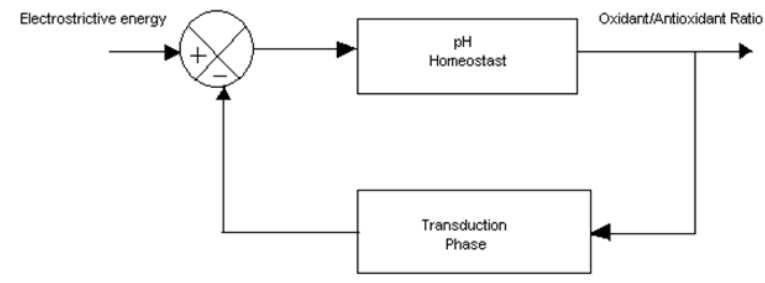

Figure 2: Block diagram of $\mathrm{pH}$ Homeostasis.

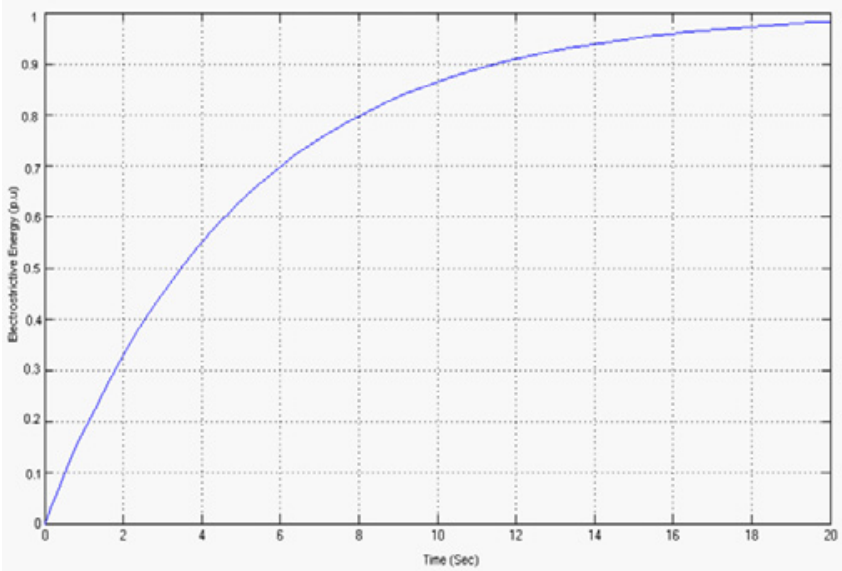

Figure 3: Electrostrictive energy input Vs Time.

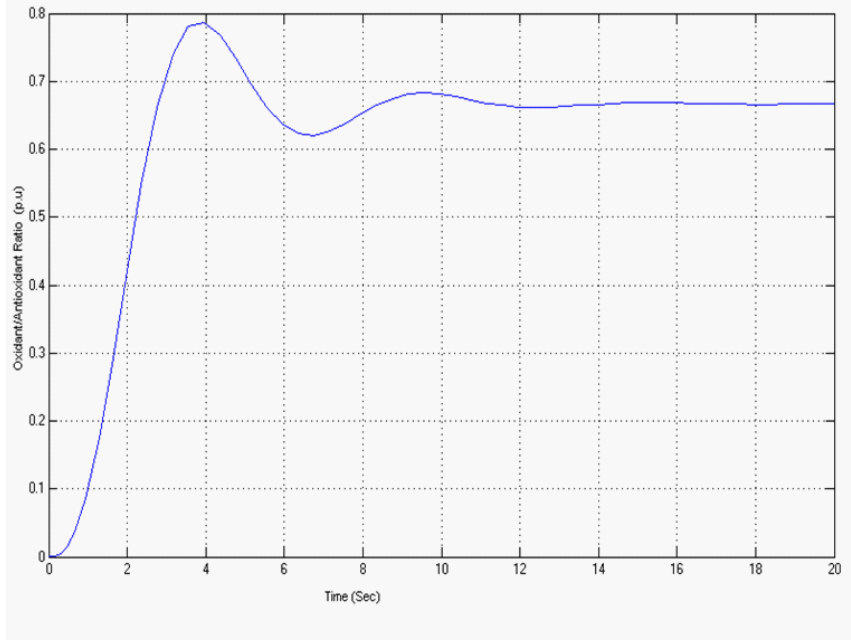

Figure 4: Oxidant/Antioxidant Ratio Vs Time.

electrostrictive energy. The block diagram of $\mathrm{pH}$ homeostasis is shown below in Figure 2:

Homeostasis is the set of processes by which constant or 'stattic' conditions are maintained within the internal environment of a subject; a homeostat is a controller involved in maintaining homeostasis $[11,17]$. The transduction phase of a subject reflects physiological changes caused by hormone release consequent on stimulation. This phase is characteristic of an individual subject [17]. The $\mathrm{pH}$ homeostat is linked with capacitance relaxation phenomena [13]. Transduction phase of this homeostat is linked with lipid peroxidation concomitant with Nrf2 (NF-E2-related factor 2) transcription factor involving the transcriptional activation of many genes $[13,18]$.

Here we will use the concept of $1^{\text {st }}$ order transfer function

$\mathrm{G}(\mathrm{S})=\frac{K}{1+\tau S}$ where $\mathrm{K}$ is the gain and $\tau$ is the time constant of the 
$1^{\text {st }}$ order system. The transfer function $G(S)$ is in complex frequency domain. The nature of electrostrictive energy input is considered as (Figure 3).

The electrostrictive energy input is regarded as exponentially rising waveform. As the transduction phase is associated with lipid peroxidation concomitant with gene transcription, this process is naturally quite slow. Therefore the transfer function of the transduction phase is taken as $1^{\text {st }}$ order system with large time constant.

The output of $\mathrm{pH}$ homeostat is given by

Figure 4 shows that with the increase of $\mathrm{pH}$, oxidant/antioxidant ratio decreases.

The block diagram of antiporter homeostasis is given in Figure 5.

Antiporter homeostasis comprises of oxidant/antioxidant ratio as input, osteoblast cell lineage as the output, antiporter homeostat and transduction phase which linked with PKC mediated pathway. The PKC mediated pathway is also a slow process. Therefore the transfer function of the antiporter transduction phase is taken as $1^{\text {st }}$ order with large time constant. The output antiporter homeostasis is given by

Figure 6 shows that osteoblast cell lineage reduces in response to decrement of oxidant/antioxidant ratio. The block diagram of PTH homeostat is given in Figure 7.

PTH homeostasis comprises of osteoblast cell lineage as input, PTH homeostat and transduction phase which is linked with PTH receptor

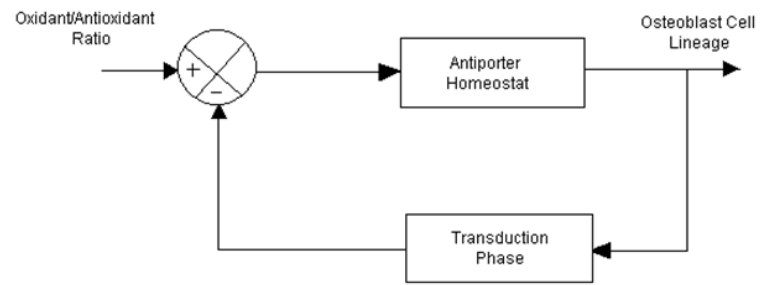

Figure 5: Block diagram of antiporter homeostasis.

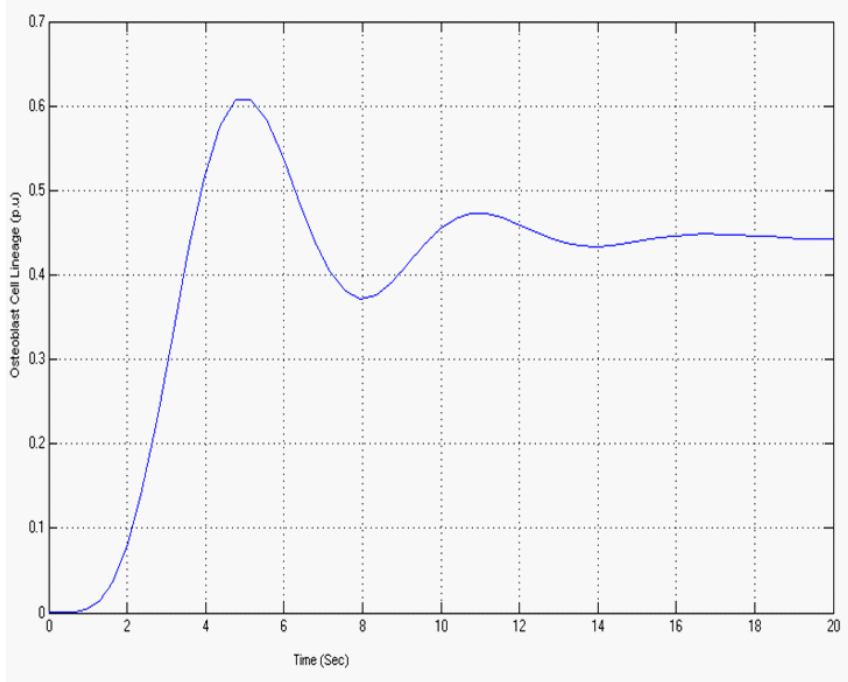

Figure 6: Osteoblast Cell Lineage (p.u) Vs Time.

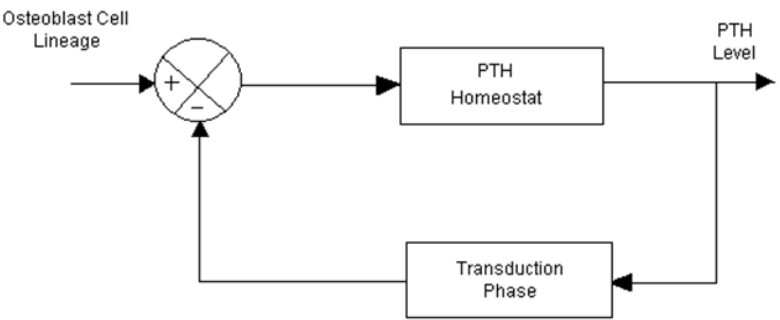

Figure 7: Block diagram of PTH homeostasis.

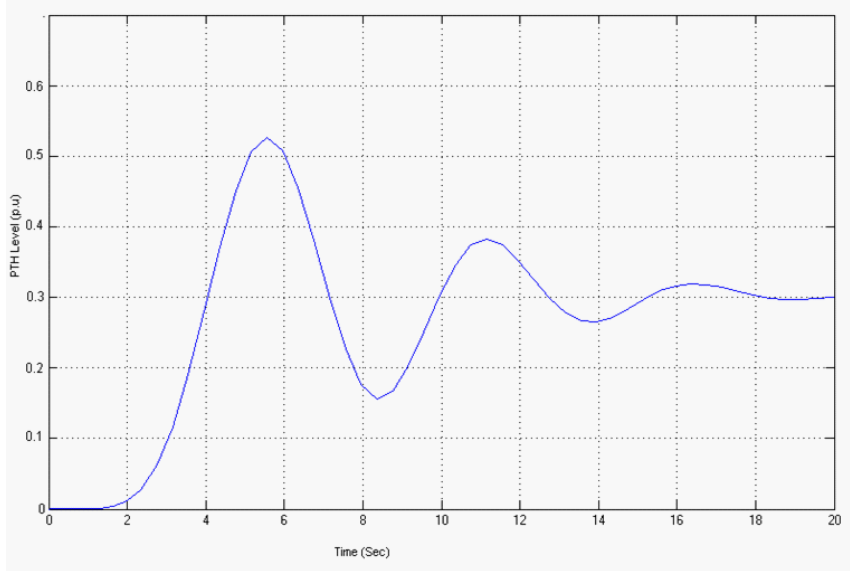

Figure 8: PTH Level Vs Time.

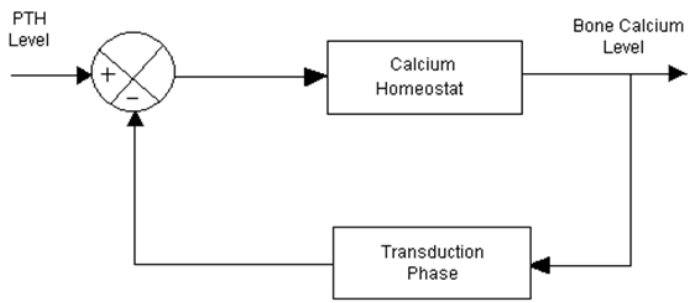

Figure 9: Block diagram of Calcium homeostasis.

coupled with cAMP response. The coupling of PTH receptor with cAMP response is moderately fast process. Therefore the transduction phase of PTH transduction phase is taken as $1^{\text {st }}$ order with medium time constant. The output of PTH homeostasis is given by the above Figure 8 shows that PTH level decreases in response to reduction of osteoblast cell lineage. The block diagram of Calcium homeostasis is given in Figure 9.

The calcium homeostasis comprises PTH level as input, calcium homeostat and transduction phase which is linked with absorption of calcium in the bone in presence of PTH receptor. The absorption of bone calcium is very fast in presence of PTH receptor. Therefore the transfer function of the transduction phase is tken as $1^{\text {st }}$ order with small time constant. The output of calcium homeostat is given in Figure 10 .

The above figure shows that bone calcium level reduces in response 
Citation: Halder S, Shankar Chakraborty A (2011) A Model of Calcium Homeostasis Linked with Electrostrictive Energy. J Health Med Informat 3:107. doi:10.4172/2157-7420.1000107

Page 5 of 5

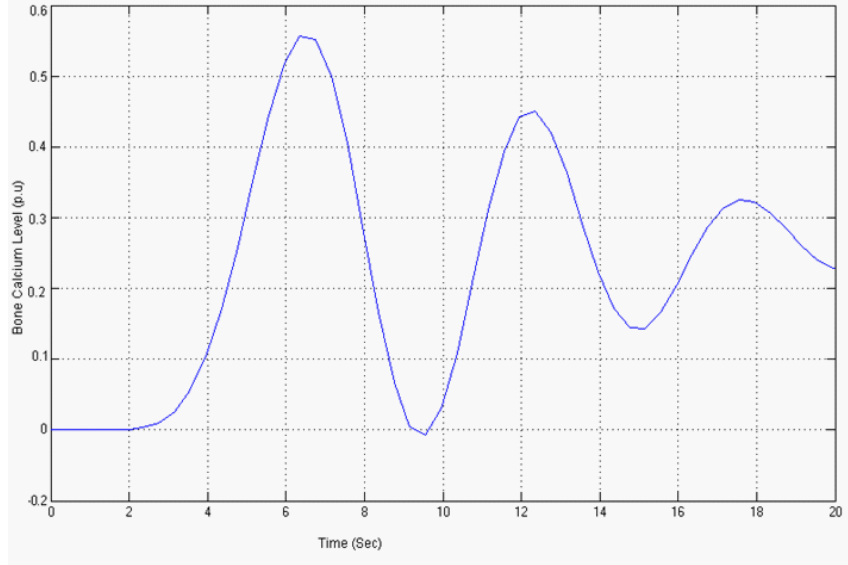

Figure 10: Bone Calcium Level Vs Time.

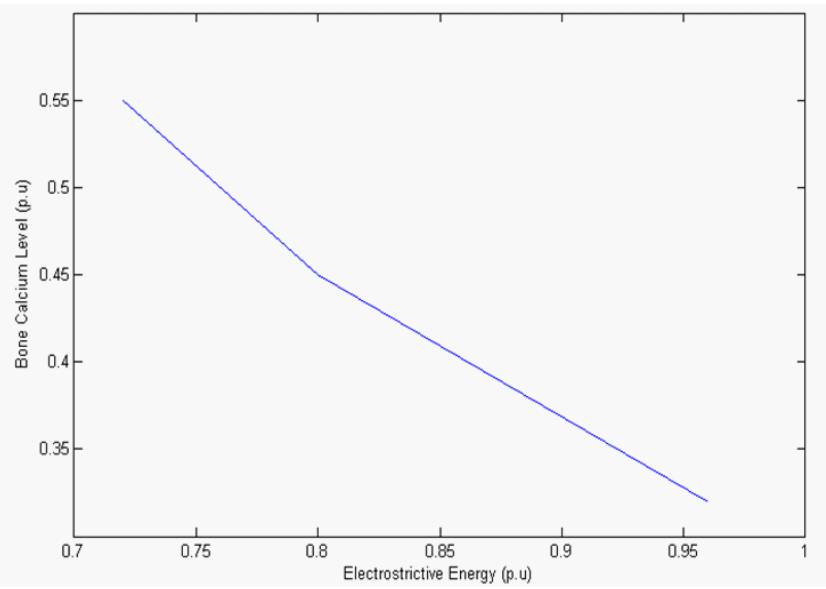

Figure 11: Bone Calcium Level (p.u) Vs Electrostrictive Energy (p.u)

to decrement of PTH level in the bone. So the bone calcium level is related with electrostrictive energy is given by following Figure 11.

\section{Conclusions}

From the above figures we can say that change in electrostrictive energy in cancer cell is responsible for reduction of oxidant/ antioxidant ratio which in turn decreases osteoblast cell lineage, which will cause decrement in PTH level which in turn reduces of bone calcium absorption. Due to the change in electrostrictive energy there is unbalance in $\mathrm{pH}$ homeostasis that will cause decrement of bone marrow stem cell production which will cause less differentiation of stem cells into osteoblast cell lineage. As a result there will less production of serum as well as bone calcium. Therefore we can come into conclusion that due to change in electrostrictive energy there will be decrement of bone calcium level.

\section{Acknowledgement}

The authors are grateful to the authorities of St. Thomas' Engg College, Kolkata. Dr. Suman Halder is particularly indebted for inspiration received from Prof. N.C.Ray of IIT Kharagpur, India and Prof. P.K.Sen of NIT Durgapur, India.

\section{References}

1. Ahlström M, Lamberg-Allardt C (1999) Regulation of Adenosine 3',5'-cyclic Monophosphate (cAMP) Accumulation in UMR-106 Osteoblast-like Cells: Role of cAMP-phosphodiesterase and cAMP Efflux. Biochem Pharmacol 58: 13351340.

2. Siddhanti R, Quarles D (1994) Molecular to pharmacologic control of osteoblast proliferation and differentiation. J Cell Biochem 55: 310-320.

3. Partridge NC, Bloch SR, Pearman AT (1994) Signal transduction pathways mediating parathyroid hormone regulation of osteoblastic gene expression. $J$ Cell Biochem 55: 321-327.

4. Jahr H, van Driel M, van Osch GJ, Weinans H, van Leeuwen JP (2005) Identification of acid-sensing ion channels in bone. Biochem Biophys Res Commun 337: 349-354.

5. Basak TK, Ramanujam T, Halder S, Goyal P, Kulshestha PM, et al. (2010) A Model Linked to E. Coli Related to Electrostrictive Energy in Cancer Cell. Sens Transd J 113: 158-166.

6. Groot TD, Lee K, Langeslag M, Xi Q, Jalink K, et al. (2009) Parathyroid Hormone Activates TRPV5 via PKADependent Phosphorylation. J Am Soc Nephrol 20: 1693-1704.

7. Graham CS, Tashjian AH Jr (1992) Mechanisms of activation of $\mathrm{Na}^{+} / \mathrm{H}^{+}$ exchange in human osteoblast-like SaOS-2 cells. Biochem J 288: 137-143.

8. Wandrup J (1989) Critical Analytical and Clinical Aspects of lonized Calcium in Neonates. Clin Chem 35: 2027-2033.

9. Basak TK, Ramanujam T, Kavitha JC, Goyal P, Garg D, et al. (2009) $\mathrm{pH}$ Homeostasis Linked with Capacitance Relaxation Phenomena and Electrostrictive Energy in Cancer Cells. Sens Transd J 109: 147-153.

10. Pliam NB, Nyiredy KO, Arnaud CD (1982) Parathyroid hormone receptors in avian bone cells. Proc Nati Acad Sci U S A 79: 2061-2063.

11. Guyton AC (2003) Textbook of Medical Physiology.

12. Feinstein TN, Wehbi VL, Ardura JA, Wheeler DS, Ferrandon S, et al. (2011) Retromer terminates the generation of cAMP by internalized PTH receptors. Nat Chem Biol 7: 278-284.

13. Pollock AS, Warnock DG, Strewler GJ (1986) Parathyroid hormone inhibition of $\mathrm{Na}^{+}-\mathrm{H}^{+}$antiporter activity in a cultured renal cell line. Am J Physiol 250: $217-$ 225

14. Lee GH, Hwang JD, Choi JY, Park HJ, Cho JY, et al. (2011) An acidic pH environment increases cell death and pro-inflammatory cytokine release in osteoblasts: The involvement of BAX Inhibitor-1. Int J Biochem Cell Biol 43 1305-1317.

15. Graham CS, Tashjian AH Jr (1992) Mechanisms of activation of $\mathrm{Na}^{+} / \mathrm{H}^{+}$ exchange in human osteoblast-like SaOS-2 cells. Biochem J 288: 137-143.

16. Fuller K, Owens JM, Chambers TJ (1998) Induction of osteoclast formation by parathyroid hormone depends on an action on stromal cells. J Endocrinol 158: 341-350.

17. Basak TK, Halder S, Kumar M, Sharma R, Midya B (2005) A topological mode of biofeedback based on catecholamine interactions. Theor Biol Med Model 2 : 11.

18. Kim SJ, Uehara H, Yazici S, Busby JE, Nakamura T, et al. (2006) Targeting platelet-derived growth factor receptor on endothelial cells of multidrug resistant prostate cancer. J Natl Cancer Inst 98: 783-793. 